\title{
Previsão de demanda por energia elétrica - Método e aplicação
}

\author{
Flávio Sanson Fogliatto (UFRGS) ffogliatto@producao.ufrgs.br \\ José Luis Duarte Ribeiro (UFRGS) ribeiro@producao.ufrgs.br \\ Liane Werner (UFRGS) liane@producao.ufrgs.br \\ Fernando de Oliveira Lemos (UFRGS) ferlemos@producao.ufrgs.br \\ Marcus Pinto de Brum (CEEE) MBrum@cee.com.br
}

\section{Resumo}

Este artigo apresenta uma metodologia para previsão de demanda por energia elétrica nas conexões da rede de distribuição de concessionárias. A sistemática de contratação de energia, por parte das concessionárias, junto às empresas transmissoras, exige a realização de previsões abrangendo extensas áreas de concessão, diversos segmentos econômicos e horários distintos. Para tanto, propõe-se a utilização de uma sistemática que combina previsões matemáticas, obtidas através de um modelo de decomposição robusto a ocorrência de eventos especiais, a opiniões de especialistas. A metodologia proposta é ilustrada através de um estudo de caso em uma distribuidora de energia elétrica da região sul do país.

Palavras chave: Previsão de demanda por energia, combinação de previsões, forecasting.

\section{Introdução}

A sistemática de contratação de energia elétrica junto às empresas transmissoras, imposta às distribuidoras pela agência que rege a operação dessas empresas, apresenta algumas particularidades desafiadoras em termos de planejamento da demanda. As concessionárias são solicitadas a declarar suas demandas trianuais nas conexões do sistema de distribuição de energia, estratificadas em demanda em horários de ponta e fora de ponta. Da projeção trianual, o primeiro ano não pode ser alterado uma vez declarado; os demais anos são sujeitos a alterações. A contratação de uma demanda menor do que o valor efetivamente realizado em uma dada conexão implica em um pagamento de multa por parte da concessionária. A contratação de uma demanda maior do que o realizado também implica em perdas financeiras para a concessionária.

A previsão de demanda por energia elétrica por parte das distribuidoras deve ser feita nas conexões do sistema de distribuição, avaliando projeções de crescimento dos setores da economia nos municípios atendidos pelas conexões. Tal previsão deve ser robusta o suficiente para não responder a eventos especiais que afetam a demanda, tal como racionamentos de energia elétrica (por exemplo, o apagão de 2000) e períodos recessivos da economia.

Este estudo consiste no desenvolvimento de uma metodologia para implementação de sistemas de previsão de demanda em distribuidoras de energia elétrica. A metodologia é fundamentada na utilização de análise estatística de séries temporais, ajustada através de opiniões de especialistas, obtidas em grupos focados.

O artigo apresenta duas contribuições relevantes. Primeiro, apresenta-se um modelo de decomposição para previsão de demanda a partir da análise de séries temporais robusto à ocorrência de eventos especiais, comuns no caso de demanda por energia elétrica. Segundo, propõe-se a combinação de previsões obtidas matematicamente e a partir de especialistas, em um procedimento estruturado que permite mapear de forma detalhada o comportamento da demanda em regiões e segmentos específicos da área de cobertura de uma dada concessionária.

O presente trabalho complementa a literatura relativamente escassa sobre previsão de demanda por energia elétrica disponível na literatura. Com relação ao modelo matemático de 
previsão, apresenta uma alternativa mais genérica e simplificada se comparada aos trabalhos de, por exemplo, Darbellay \& Slama (2000) e Harris \& Liu (1993). Com relação ao uso de combinação de previsões, complementa e amplia o método proposto por Anderson (1995).

\section{Referencial Teórico}

Previsões de demanda desempenham um importante papel em diversas áreas na gestão de organizações; por exemplo, na área financeira (no planejamento de recursos) e na área de recursos humanos (no planejamento de modificações no nível da força de trabalho). Previsões são também essenciais na operacionalização de diversos aspectos do gerenciamento de operações.

Previsões de demanda são elaboradas utilizando técnicas quantitativas, qualitativas ou combinações de ambas. Técnicas quantitativas (ou técnicas de forecasting) baseiam-se na análise de séries temporais (dados que descrevem a variação da demanda ao longo do tempo). Dentre elas, encontram-se a decomposição de séries temporais, o alisamento exponencial e a metodologia de Box-Jenkins. Mais recentemente, redes neurais passaram também a ser utilizadas para esse fim (ARMSTRONG, 2001).

A decomposição de séries temporais é um estudo descritivo, onde a série é decomposta em quatro componentes. $\mathrm{O} 1^{\circ}$ componente é a tendência, que verifica o sentido de deslocamento da série ao longo do tempo; o $2^{\circ}$ componente é o ciclo, movimento ondulatório que ao longo de vários anos tende a ser periódico; o $3^{\circ}$ componente é a sazonalidade, que tem a duração de curto prazo, inferior a um ano; o $4^{\circ}$ componente são acontecimentos aleatórios de dificíl modelagem (MAKRIDAKIS et al., 1998).

Nos modelos de suavização exponencial, as observações mais recentes na série temporal recebem maior importância para a estruturação do modelo. Para analisar séries que apresentam tendência, pode-se utilizar a modelagem biparamétrica de Holt; já para séries que apresentam sazonalidade é indicado o modelo de suavização exponencial de Holt-Winters (MAKRIDAKIS et al., 1998).

Os modelos de Box-Jenkins são construídos com base na estrutura de autocorrelação da série temporal e subdividem-se em: (i) modelos autoregressivos, que exploram a estrutura de autocorrelação da variável; (ii) modelos de médias móveis, que exploram a estrutura de autocorrelação dos erros de previsão; (iii) modelos mistos autoregressivos e de médias móveis, adequados para séries onde é desejável um número não muito grande de parâmetros; e (iv) modelos mistos autoregressivos integrados e de médias móveis, para séries não-estacionárias, sendo necessário realizar diferenças do valor da variável com o valor da variável em períodos anteriores (BOX et al., 1994).

As técnicas qualitativas baseiam-se em opiniões de especialistas, sendo vulneráveis a tendências que podem comprometer a confiabilidade de seus resultados. Técnicas qualitativas têm sido, historicamente, as mais utilizadas na previsão da demanda (MENTZER \& COX, 1997); dentre elas, destacam-se o método Delphi e os grupos focados.

Em linhas gerais, no método Delphi consulta-se um grupo de especialistas a respeito de eventos futuros através de questionários. O princípio do método é intuitivo e iterativo, onde os resultados de cada fase são analisados. A síntese dos resultados é repassada continuadas vezes até que seja obtida uma convergência das respostas (WRIGHT \& GIOVINAZZO, 2000). Para Patton (1987), grupo focado é uma entrevista com um pequeno grupo de pessoas sobre um tópico específico. Os participantes formam um grupo relativamente homogêneo e são convidados a refletir sobre uma questão proposta. Os participantes escutam as respostas dos outros participantes e fazem comentários adicionais, além de suas próprias respostas. O grupo em geral, é composto por seis a oito pessoas, que participam de uma sessão com duração de uma hora a duas horas. 
Técnicas qualitativas costumam apresentar um baixo grau de precisão; apesar disto, continuam sendo amplamente utilizadas nas empresas, mesmo com a difusão de técnicas de previsão mais avançadas, impulsionada pelo avanço na capacidade de processamento e armazenamento de dados computacionais (SANDERS \& MANRODT, 1994). A extensa utilização das técnicas qualitativas parece estar relacionada ao fato das previsões por elas geradas corresponderem às metas de demanda estabelecidas pelas empresas (DIAS, 1999).

A prática usual é, dadas duas ou três previsões do mesmo evento, determinar a melhor delas através de alguma medida de acurácia. A melhor previsão é, então, aproveitada, e as outras, descartadas. Através do descarte de previsões classificadas como inferiores, muitas vezes alguma informação útil é perdida. Logo, ao invés de escolher uma única técnica de previsão, parece razoável considerar informações provenientes de várias técnicas e combiná-las.

A combinação é uma abordagem atraente para realizar previsões, visto que, ao invés de tentar determinar a melhor técnica, formula-se o problema questionando quais técnicas poderiam ajudar na melhoria da acurácia. Como as previsões podem ser afetadas por diversos fatores, cada técnica pode contribuir capturando algum tipo de informação que influencia esses fatores (CLEMEN, 1989).

A combinação de técnicas quantitativas e qualitativas pode ser descrita da seguinte maneira: primeiramente, gera-se, com base em dados históricos, um modelo; após, uma previsão quantitativa. Paralelamente, realiza-se uma análise subjetiva dos dados históricos, agregando informações contextuais, de onde se obtém uma previsão qualitativa. Estas previsões são, então, combinadas, gerando a previsão final (WEBBY \& O'CONNOR, 1996).

Flores \& White (1988) propõem uma estrutura para combinação de previsões que estabelece duas dimensões: $(i)$ seleção das técnicas de previsão-base, onde define-se quais previsões incluir na combinação, isto é, selecionam-se as técnicas que irão participar da combinação; e (ii) seleção do método de combinação, onde define-se a forma de combinação das técnicas. Segundo Clemen (1989), métodos têm sido desenvolvidos para encontrar a melhor combinação de previsões e o resultado tem sido unânime: combinar previsões conduz ao aumento de acurácia da previsão (combinada) em relação a qualquer previsão individual.

\section{Método proposto}

O método para previsão de demanda por energia elétrica proposto é baseado em um modelo estatístico que captura o padrão de variação de consumo de energia e incorpora a opinião de especialistas como elemento calibrador, refinando as previsões derivadas do modelo. O método gera previsões de consumo de energia por município em uma área de concessão de interesse, a qual é convertida posteriormente em demanda (carga) em conexões, sendo esse o resultado de interesse obtido a partir do sistema de previsão.

Com a utilização do método proposto pode-se gerar previsões de demanda por energia, dadas na forma de consumo e/ou carga prevista, estratificada conforme região de consumo, município, horário, mês, dia da semana e classe de consumidor, dependendo da necessidade do tomador de decisão. A Figura 1 apresenta o sistema de previsão de uma forma geral. Os detalhes de cada passo para obtenção da previsão final são apresentados na sequência. Em paralelo ao detalhamento do sistema de previsão, apresenta-se uma aplicação do método. $\mathrm{O}$ estudo de caso reportado ocorreu em uma distribuidora de energia elétrica do RS. A distribuidora tem a concessão de uma região composta por 7 gerências (Bagé, Camaquã, Osório, Pelotas, Porto Alegre, Rio Grande e São Jerônimo).

A primeira etapa do método é a de definição do problema a ser resolvido. O problema a ser resolvido pelo sistema de previsão proposto é a obtenção da previsão mensal de carga nos horários de ponta (carga pesada) e fora de ponta (carga média e leve) nos pontos de conexão da 
área de concessão da empresa. O sistema de previsão visa auxiliar no planejamento de contratação do uso do Sistema de Transmissão (carga nas conexões) por empresas distribuidoras junto à Rede Básica e no planejamento de expansão do sistema de distribuição em curto, médio e longo prazo. A contratação do uso do Sistema de Transmissão contempla 3 anos, comprometendo as empresas distribuidoras com a expansão do sistema.

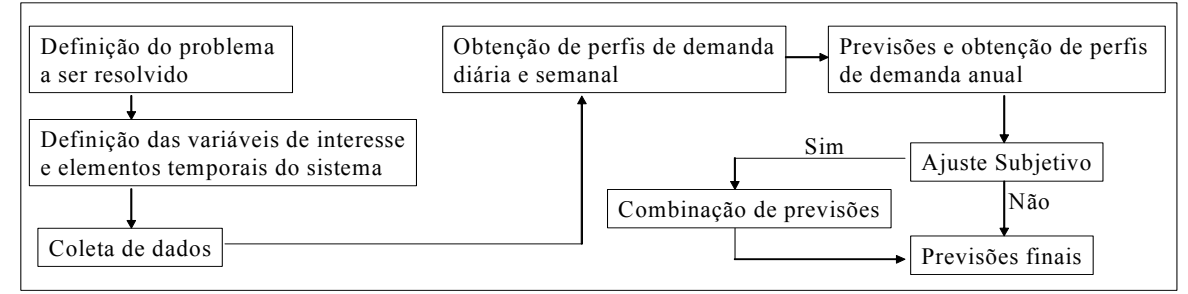

Figura 1 - Método proposto para previsão de demanda por energia elétrica

A demanda (carga) nas conexões é o agregado das demandas medidas nos alimentadores que compõem as conexões. Assim, além da demanda agregada por conexão, é necessária a análise da demanda desagregada nos alimentadores. Devem também ser analisadas variáveis subjetivas com efeito potencial sobre a demanda. No caso em tela, tais variáveis foram mensuradas a partir de estudos em grupos focados com especialistas da área comercial da empresa. O objetivo é identificar os perfis de crescimento das classes de consumidores industrial, comercial, rural, outros e residencial na região de concessão ( 7 gerências). Tais perfis devem ser incorporados nas projeções de demanda quantitativa.

A segunda etapa do método proposto é a de definição das variáveis de interesse e elementos temporais do sistema. No estudo de caso, definiu-se como variáveis de interesse o consumo de energia por município e classe de consumidores (para obtenção das previsões de consumo e coeficientes de sazonalidade anual) e a demanda de energia (carga) por gerência (para obtenção de coeficientes de sazonalidade diária e semanal).

Os elementos temporais do sistema também devem ser definidos. O período de previsão foi definido como mensal, já que os dados de consumo que alimentam o sistema são dados nesta base. O horizonte de previsão foi definido em 3 anos devido ao período de contratação de demanda junto ao Sistema de Transmissão. $O$ intervalo de previsão depende da necessidade de consulta do tomador de decisão.

A terceira etapa é a de coleta de dados e obtenção de perfis de demanda. Os dados de entrada do sistema de previsão proposto são: $(i)$ dados de consumo histórico dos municípios; (ii) mapeamento do relacionamento entre município, alimentador, subestação e conexão; e (iii) dados de demanda por região (gerência). Verificou-se, então, a disponibilidade dos dados e a confiabilidade dos mesmos.

Os dados de consumo coletados geraram séries históricas por alimentador, com início em janeiro de 1997 e fim em abril de 2004. Os dados de consumo são dados faturados que podem ser ajustados devido a perdas do sistema (perdas técnicas e de faturamento), sendo o percentual de perda variável por município e classe.

A previsão inicial e ajuste subjetivo se dão na estimativa de consumo de energia por município, mas as séries temporais utilizadas no sistema são de consumo de alimentadores, que podem estar vinculados a mais de um município. Então é necessário um trabalho de composição dos históricos dos municípios através da desagregação do consumo dos alimentadores ligados a ele. A Tabela 1 exemplifica a composição do histórico de municípios vinculados a um alimentador (R.COL/EST.B/L), informando, por exemplo, que 3,73\% do consumo residencial do alimentador refere-se ao consumo residencial de Bagé. 


\begin{tabular}{lccccc}
\hline & \multicolumn{5}{c}{ \% de Consumo do Alimentador R.COL/EST.B/L } \\
\hline MUNICÍPIO/CLASSE & RESIDENCIAL & COMERCIAL & INDUSTRIAL & RURAL & OUTROS \\
\hline BAGÉ & $3,73 \%$ & $47,78 \%$ & $0,00 \%$ & $9,86 \%$ & $0,00 \%$ \\
DOM PEDRITO & $4,57 \%$ & $0,46 \%$ & $0,00 \%$ & $36,57 \%$ & $0,00 \%$ \\
LAVRAS DO SUL & $91,70 \%$ & $51,76 \%$ & $100,00 \%$ & $53,58 \%$ & $100,00 \%$ \\
\hline Total & $100,00 \%$ & $100,00 \%$ & $100,00 \%$ & $100,00 \%$ & $100,00 \%$ \\
\hline
\end{tabular}

Tabela 1 - Percentuais de composição da série histórica de municípios para um alimentador

Um município pode estar vinculado a mais de um alimentador e este pode estar ligado a mais de uma conexão. Assim, faz-se necessário um mapeamento, pois a previsão por município (por classe de consumidor) deve ser desagregada por alimentador para então compor a previsão por conexão. Os percentuais de rateamento das previsões orientarão as composições de previsão em alimentadores e conexões.

Os dados de demanda por gerência auxiliam a obtenção dos perfís de sazonalidade diária e semanal. Os dados de demanda utilizados foram obtidos de leituras minuto a minuto dos últimos doze meses (maio de 2003 à abril de 2004) e integrados por hora para cada conexão das gerências. Calculou-se a média das demandas nas conexões para obter a sazonalidade diária (coeficientes horários). Os dados também foram agregados por dia, sendo calculada a média para as conexões para obtenção da sazonalidade semanal (coeficientes diários). Os resultados são exemplificados na Tabela 2.

\begin{tabular}{ccccccccccccc}
\hline Hora & $\mathbf{1}$ & $\mathbf{2}$ & $\mathbf{3}$ & $\mathbf{4}$ & $\mathbf{5}$ & $\mathbf{6}$ & $\mathbf{7}$ & $\mathbf{8}$ & $\mathbf{9}$ & $\mathbf{1 0}$ & $\mathbf{1 1}$ & $\mathbf{1 2}$ \\
\hline média & 15,77 & 14,97 & 14,70 & 14,39 & 14,96 & 15,54 & 16,36 & 17,44 & 18,52 & 19,10 & 19,41 & 17,74 \\
coef. & 0,88 & 0,83 & 0,82 & 0,80 & 0,83 & 0,87 & 0,91 & 0,97 & 1,03 & 1,07 & 1,08 & 0,99 \\
\hline Hora & $\mathbf{1 3}$ & $\mathbf{1 4}$ & $\mathbf{1 5}$ & $\mathbf{1 6}$ & $\mathbf{1 7}$ & $\mathbf{1 8}$ & $\mathbf{1 9}$ & $\mathbf{2 0}$ & $\mathbf{2 1}$ & $\mathbf{2 2}$ & $\mathbf{2 3}$ & $\mathbf{2 4}$ \\
\hline média & 17,68 & 18,53 & 18,73 & 18,91 & 18,84 & 19,31 & 20,38 & 20,47 & 21,77 & 20,91 & 18,83 & 17,01 \\
coef. & 0,99 & 1,03 & 1,05 & 1,05 & 1,05 & 1,08 & 1,14 & 1,14 & 1,21 & 1,17 & 1,05 & 0,95 \\
\hline
\end{tabular}

\begin{tabular}{lcccc}
\hline Dia & Seg & Ter & Qua & Qui \\
\hline média & 17,79 & 18,86 & 18,90 & 19,02 \\
coef. & 0,99 & 1,05 & 1,05 & 1,06 \\
\hline Dia & Sex & Sab & Dom & \\
\hline média & 18,34 & 18,12 & 15,01 & \\
coef. & 1,02 & 1,01 & 0,83 & \\
\hline
\end{tabular}

Tabela 2 - Esquerda: demanda média e coeficientes horários de sazonalidade diária para Gerência de Bagé; direita: Demanda média e coeficientes diários de sazonalidade semanal para Gerência de Bagé

Os coeficientes horários e diários flexibilizam a obtenção de previsões de demanda de energia. A previsão pode ser estratificada conforme necessidades dos tomadores de decisão, como, por exemplo, previsões para carga no horário de ponta (das 18 às $21 \mathrm{~h}$ ) em dias úteis ou carga no horário fora de ponta (das 21 às 18h) em dias úteis, no período de Ponta Úmida (maio a novembro).

$\mathrm{Na}$ quarta etapa faz-se a previsão de consumo de energia e obtenção dos coeficientes de sazonalidade anual. A previsão matemática do consumo (por município) utiliza um modelo de decomposição multiplicativo de previsão, contendo três termos [eq. (1)].

Consumo no mês $j=$ Consumo atual $\times$ Sazonalidade do mês $j \times$ Taxa de crescimento

O termo "consumo atual" para um determinado município é obtido através de uma média móvel dos 6 últimos meses. A sazonalidade do mês $j$ é obtida comparando a sazonalidade do próximo mês com a sazonalidade dos meses incluídos na média móvel [eq. (2)].

Sazonalidade mês $j=$ Coef. Sazon. mês $j$ / Coef. Sazon. dos meses da média móvel

O coeficiente de sazonalidade do mês $j$ é calculado comparando o consumo médio observado em todos os meses $j$ da série com o consumo médio observado na série [eq. (3)]. A variável $n$ denota o tamanho da série temporal.

Coef. Sazon. mês $j=$ Consumo médio nos meses $j$ / Consumo médio nos meses 0 a $n-5$

O coeficiente de sazonalidade dos meses da média móvel é calculado comparando o consumo médio observado nos períodos correspondentes aos meses da média móvel (MM) com o consumo médio observado em todo o período [eq. (4)].

Coef. Sazon. MM = Cons.médio dos períodos dos meses da MM / Cons.médio nos $n$ meses (4) A taxa de crescimento mensal do consumo (TC) é calculada comparando o consumo médio dos últimos 12 meses com o consumo médio dos primeiros 12 meses da série histórica de 
consumo do município analisado [eq. (5)]. O procedimento se apóia nos primeiros 12 meses da série histórica para evitar os efeitos de eventos especiais (ex.: apagão) sobre o consumo.

$\mathrm{TC}=(\text { Cons.médio nos últimos } 12 \text { meses /Cons.médio nos primeiros } 12 \text { meses })^{(1 /(n-12))}$

A previsão de consumo para um mês $j$ é convertida em demanda através de um cálculo multiplicativo. A demanda de energia é um valor pontual de unidade KW. Como a unidade de consumo é KWh e o valor previsto corresponde a um valor mensal, converte-se o consumo dividindo o mesmo pelos dias do mês $j$ e pelas horas do dia (24 horas). A Tabela 3 apresenta os resultados da aplicação do método de decomposição multiplicativa proposto para o município de Bagé, Classe Residencial. Para exemplificar, só foram apresentadas previsões no horizonte de 6 meses. O método gera como resultados: (i) o consumo atual; (ii) um crescimento anual (TC); (iii) um perfil de sazonalidade anual (coeficientes de sazonalidade mensais); e (iv) previsões mensais de demanda (carga).

\begin{tabular}{|c|c|c|c|c|c|c|c|c|c|c|c|c|}
\hline Consumo atual & 4.958 & KWh & & & & Cresciment & inual & onsumo & $-1,0 \%$ & & & \\
\hline Sazonalidade / Mês & jan & fev & mar & $\overline{a b r}$ & mai & jun & jul & ago & set & out & nov & dez \\
\hline Coef. Matemático & 1,08 & 0,98 & 1,04 & 1,04 & 1,00 & 1,01 & 1,03 & 1,01 & 0,99 & 0,94 & 0,96 & 0,94 \\
\hline $\begin{array}{l}\text { Previsão de } \\
\text { demanda }(K W)\end{array}$ & $\begin{array}{c}\text { mai-04 } \\
5.016,42\end{array}$ & $\begin{array}{c}\text { jun-04 } \\
4.810,40\end{array}$ & $\begin{array}{c}\text { jul-04 } \\
4.836,04\end{array}$ & $\begin{array}{c}\text { ago-04 } \\
4.858,74\end{array}$ & $\begin{array}{c}\text { set-04 } \\
4.912,56\end{array}$ & $\begin{array}{cc} & \text { out-04 } \\
6 & 4.641,45\end{array}$ & & & & & & \\
\hline
\end{tabular}

Tabela 3 - Resultados obtidos com a aplicação do método (Bagé - Classe Residencial)

$\mathrm{Na}$ etapa de ajuste das previsões pelos especialistas, a previsão matemática é corrigida utilizando a opinião de especialistas. Este ajuste é necessário pois as previsões refletem o histórico de consumo e demanda, mas não eventuais mudanças de mercado ou expansões futuras. Os especialistas validaram as previsões obtidas com o modelo matemático e fizeram inferências baseadas nas informações de expansão ou retração na demanda de cada município. Os especialistas também corrigiram os perfis de sazonalidade, em função de eventos que existiam (ou não) no passado e deixarão de (ou passarão a) existir.

A opinião dos especialistas foi obtida através de Grupos Focados, onde em geral participaram mais de 3 especialistas. Para cada município, os especialistas avaliaram dois aspectos: $(i)$ os coeficientes de sazonalidade, apresentados na forma de perfil anual, e (ii) a taxa de crescimento, apresentada na forma de crescimento anual (\%). Para cada gerência, os especialistas devem avaliar os coeficientes de sazonalidade apresentados na forma de perfis semanais e diários, e ajustá-los por classe de cliente. Os especialistas foram questionados também sobre a certeza acerca dos ajustes sugeridos (confiança do ajuste dos especialistas). Os parâmetros fornecidos pelos especialistas serão combinados com os parâmetros matemáticos, proporcionalmente à confiança que existe em cada fonte de dados.

Os ajustes dos coeficientes de sazonalidade foram auxiliados por análises gráficas dos perfis para facilitar a visualização do comportamento da demanda. A Figura 2 exemplifica o ajuste nos coeficientes de sazonalidade diário para a classe residencial da Gerência de Bagé. Os ajustes dos especialistas nos coeficientes mensais de sazonalidade anual e taxa de crescimento para a classe residencial do município Bagé podem ser vistos na Tabela 4.

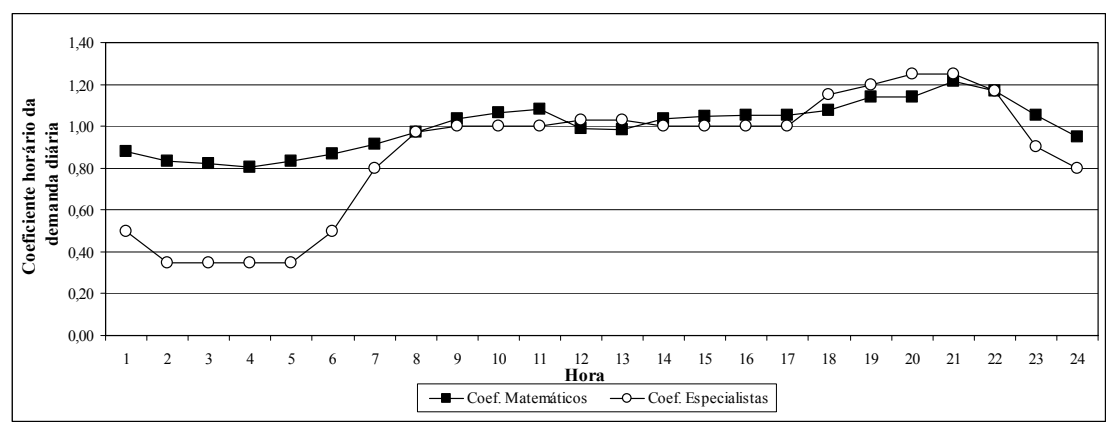

Figura 2 - Ajustes subjetivos nos coeficientes de sazonalidade diário para Gerência de Bagé (Residencial) 


\begin{tabular}{|c|c|c|c|c|c|c|c|c|c|c|c|c|}
\hline \multicolumn{13}{|c|}{ Crescimento anual de consumo } \\
\hline \multicolumn{2}{|c|}{ Matemático $-1,0 \%$} & \multicolumn{4}{|c|}{ Especialistas $0,0 \%$} & & & \multicolumn{5}{|c|}{ Confiança Especialistas $90,0 \%$} \\
\hline \multicolumn{13}{|c|}{ Sazonalidade } \\
\hline Mês & jan & fev & mar & abr & mai & jun & jul & ago & set & out & nov & dez \\
\hline Coef. Matemático & 1,08 & 0,98 & 1,04 & 1,04 & 1,00 & 1,01 & 1,03 & 1,01 & 0,99 & 0,94 & 0,96 & 0,94 \\
\hline Coef. Especialistas & 1,05 & 1,00 & 1,05 & 1,05 & 1,05 & 1,10 & 1,10 & 1,01 & 0,99 & 0,94 & 0,96 & 0,94 \\
\hline Confiança Especialistas & 0,80 & & & & & & & & & & & \\
\hline
\end{tabular}

Tabela 4 - Ajustes subjetivos nos coeficientes de sazonalidade e taxa de crescimento anuais (Bagé-Residencial) Após os ajustes dos especialistas, tem-se a etapa de combinação das previsões, onde obtêm-se combinações ponderadas das estimativas de tendência e coeficientes de sazonalidade. A confiança dos ajustes dos especialistas (CE) é usada como peso para a ponderação dos valores matemáticos e dos especialistas. As Tabelas 5 e 6 apresentam as variáveis ponderadas.

\begin{tabular}{lcccccccccccc}
\hline \multicolumn{10}{c}{ Taxa de crescimento de consumo } \\
\hline \multicolumn{1}{c}{} & \multicolumn{1}{c}{ Especialistas $0,0 \%$} & \multicolumn{1}{c}{ Crescimento ponderado $-0,1 \%$} \\
\hline & Matemática & $-1,0 \%$ & \multicolumn{1}{c}{ Sazonalidade } & & & & & \\
\hline Mês & jan & fev & mar & abr & mai & jun & jul & ago & set & out & nov & dez \\
\hline Coef. Matemático & 1,08 & 0,98 & 1,04 & 1,04 & 1,00 & 1,01 & 1,03 & 1,01 & 0,99 & 0,94 & 0,96 & 0,94 \\
Coef. Especialistas & 1,05 & 1,00 & 1,05 & 1,05 & 1,05 & 1,10 & 1,10 & 1,01 & 0,99 & 0,94 & 0,96 & 0,94 \\
Coef. Ponderado & 1,06 & 1,00 & 1,05 & 1,05 & 1,04 & 1,08 & 1,09 & 1,01 & 0,99 & 0,94 & 0,96 & 0,94 \\
\hline
\end{tabular}

Tabela 5 - Combinação de taxa de crescimento de consumo e dos coeficientes de sazonalidade anual (BagéResidencial)

\begin{tabular}{lcccccccccccc}
\hline Hora & $\mathbf{1}$ & $\mathbf{2}$ & $\mathbf{3}$ & $\mathbf{4}$ & $\mathbf{5}$ & $\mathbf{6}$ & $\mathbf{7}$ & $\mathbf{8}$ & $\mathbf{9}$ & $\mathbf{1 0}$ & $\mathbf{1 1}$ & $\mathbf{1 2}$ \\
\hline Coef. Matemático & 0,88 & 0,83 & 0,82 & 0,80 & 0,83 & 0,87 & 0,91 & 0,97 & 1,03 & 1,07 & 1,08 & 0,99 \\
Coef. Especialistas & 0,50 & 0,35 & 0,35 & 0,35 & 0,35 & 0,50 & 0,80 & 0,97 & 1,00 & 1,00 & 1,00 & 1,03 \\
Coef. Ponderado & 0,54 & 0,40 & 0,40 & 0,40 & 0,40 & 0,54 & 0,81 & 0,97 & 1,00 & 1,01 & 1,01 & 1,03 \\
\hline Hora & $\mathbf{1 3}$ & $\mathbf{1 4}$ & $\mathbf{1 5}$ & $\mathbf{1 6}$ & $\mathbf{1 7}$ & $\mathbf{1 8}$ & $\mathbf{1 9}$ & $\mathbf{2 0}$ & $\mathbf{2 1}$ & $\mathbf{2 2}$ & $\mathbf{2 3}$ & $\mathbf{2 4}$ \\
\hline Coef. Matemático & 0,99 & 1,03 & 1,05 & 1,05 & 1,05 & 1,08 & 1,14 & 1,14 & 1,21 & 1,17 & 1,05 & 0,95 \\
Coef. Especialistas & 1,03 & 1,00 & 1,00 & 1,00 & 1,00 & 1,15 & 1,20 & 1,25 & 1,25 & 1,17 & 0,90 & 0,80 \\
Coef. Ponderado & 1,03 & 1,00 & 1,00 & 1,01 & 1,01 & 1,14 & 1,19 & 1,24 & 1,25 & 1,17 & 0,92 & 0,81 \\
\hline
\end{tabular}

\begin{tabular}{lcccc}
\hline Dia & Seg & Ter & Qua & Qui \\
\hline Coef. Matemático & 0,99 & 1,05 & 1,05 & 1,06 \\
Coef. Especialistas & 0,99 & 1,00 & 1,00 & 1,02 \\
Coef. Ponderado & 0,99 & 1,00 & 1,00 & 1,02 \\
\hline Dia & Sex & Sab & Dom & \\
\hline Coef. Matemático & 1,02 & 1,01 & 0,83 & \\
Coef. Especialistas & 1,02 & 1,10 & 1,10 & \\
Coef. Ponderado & 1,02 & 1,09 & 1,07 & \\
\hline
\end{tabular}

Tabela 6 - Esquerda: combinação dos coeficientes de sazonalidade diária (Bagé-Residencial); direita: combinação dos coeficientes de sazonalidade semanal (Bagé-Residencial)

A última etapa do método é a de previsão final. Como o objetivo é a previsão na conexão, a previsão do município é rateada por conexão e então são aplicados os coeficientes de sazonalidade diária e semanal. A Tabela 7 apresenta as previsões correspondentes a conexão Bagé 2 (BAG2).

\begin{tabular}{|c|c|c|c|c|c|c|}
\hline \multirow[t]{2}{*}{ Município } & \multicolumn{5}{|c|}{ Previsão para outubro de 2004 (KW) } & \multirow[b]{2}{*}{ Total } \\
\hline & Residencial & Comercial & Industrial & Rural & Outros & \\
\hline Bagé & 4.641 & 1.677 & 2.684 & 1.639 & 691 & 11.332 \\
\hline Candiota & 1.181 & 351 & 5.034 & 553 & 250 & 7.369 \\
\hline Dom Pedrito & 1.493 & 447 & 1.514 & 890 & 258 & 4.602 \\
\hline Hulha Negra & 15 & 23 & 735 & 1.032 & 11 & 1.816 \\
\hline Lavras do Sul & 314 & 91 & 22 & 396 & 90 & 913 \\
\hline Pinheiro Machado & 886 & 250 & 2.917 & 412 & 199 & 4.664 \\
\hline
\end{tabular}

Tabela 7 - Previsões de demanda para os municípios vinculados a conexão BAG2

\begin{tabular}{|c|c|c|c|c|c|c|c|}
\hline Hora & & 1 & 2 & $\ldots$ & 24 & & \\
\hline Coeficiente horário & & 0,54 & 0,40 & $\ldots$ & 0,81 & & \\
\hline Total $=3.0696 \mathrm{KW}$ & & 16.513 & 12.232 & $\ldots$ & 25.013 & Ponta & Fora de Ponta \\
\hline \multirow{8}{*}{ Coeficiente diário } & seg 0,99 & 16.315 & 12.086 & $\ldots$ & 24.714 & 37.803 & 35.373 \\
\hline & ter 1,00 & 16.591 & 12.290 & $\ldots$ & 25.132 & 38.442 & 35.970 \\
\hline & qua 1,00 & 16.595 & 12.293 & $\ldots$ & 25.137 & 38.451 & 35.978 \\
\hline & qui 1,02 & 16.903 & 12.522 & $\ldots$ & 25.605 & 39.166 & 36.648 \\
\hline & set 1,02 & 16.822 & 12.461 & $\ldots$ & 25.481 & 38.977 & 36.471 \\
\hline & sab 1,09 & 18.009 & 13.341 & $\ldots$ & 27.281 & 41.729 & 39.046 \\
\hline & dom 1,07 & 17.725 & 13.130 & $\ldots$ & 26.849 & 41.069 & 38.428 \\
\hline & & & & & visão(KW) & 41.729 & 39.046 \\
\hline
\end{tabular}

Tabela 8 - Previsões de demanda para a conexão BAG2

A Tabela 8 exemplifica a obtenção da previsão para a conexão Bagé 2 (BAG2) para os horários de ponta (das 18 às $21 \mathrm{~h}$ ) e fora de ponta ( $22 \mathrm{~h}$ às $17 \mathrm{~h}$ ) no mês de outubro (período de Ponta Úmida). Como o modelo é multiplicativo, basta multiplicar a previsão total dos 
município (30.696KW) pelos coeficientes de sazonalidade diária e semanal. As colunas Ponta e Fora de Ponta apresentam o valor máximo para os horários e dias correspondentes. A demanda utilizada como previsão é o maior valor estimado para os dois horários. Para a conexão BAG2, a previsão para ponta é de 41,73MW e, para fora de ponta, de 39,05MW.

\section{Conclusão}

Neste artigo apresentou-se uma metodologia para obtenção de previsões de demanda por energia elétrica. $\mathrm{O}$ método foi desenvolvido motivado pela atual sistemática de contratação de energia imposta às concessionárias. Nela, concessionárias são solicitadas a pré-contratar a demanda por energia em um período de três anos, dos quais o mais recente está sujeito a revisões e ajustes.

O modelo matemático proposto no método, baseado em modelos de decomposição, gerou previsões consistentes em quase todos os casos analisados na aplicação prática. Previsões inconsistentes foram observadas apenas em municípios onde o fornecimento de energia iniciou durante o período de análise (após 1997); nesses casos, a taxa de crescimento resulta claramente superdimensionada. Para solucionar esse problema há duas alternativas: $(i)$ utilizar dados de demanda histórica apenas após o amadurecimento do fornecimento de energia elétrica; ou (ii) informar a taxa de crescimento a partir do conhecimento de especialistas. Em todos os casos, o modelo matemático, combinado a informações subjetivas obtidas de especialistas, mostrou-se flexível à necessidade de informações do tomador de decisão.

\section{Referências}

ANDERSON, E.A. (1995) - Judgmental and statistical methods of peak electricity load management. Int.J.Forecasting, v.11, 295-305.

ARMSTRONG, J.S. (2001) - Principles of Forecasting. Kluwer Academic Publishers.

DARBELLAY, G.A. \& SLAMA, M. (2000) - Forecasting the short-term demand for electricity. Int.J.Forecasting, v.16, 71-83.

BOX, G.E.P.; JENKINS, G.M. \& REINSEL, G.C. (1994) - Time series analysis - forecasting and control, $3^{\mathrm{a}}$ ed., Prentice Hall, New Jersey.

CLEMEN, R.T. (1989) - Combining Forecasts: A Review and Annotated Bibliography. Int.J.Forecasting, v.5, p.559-583.

DIAS, G.P.P. (1999) - Proposta de processo de previsão de vendas para bens de consumo. Anais do XIX ENEGEP - CD-ROM, Rio de Janeiro.

FLORES, B.E. \& WHITE, E.M. (1988) - A Framework for the Combination of Forecasts. Journal Academic Marketing Science, v.16 (3-4), p.95-103.

HARRIS, J.L. \& LIU, L. (1993)- Dynamic structural analysis and forecasting of residential electricity consumption. Int.J.Forecasting, v.9, 437-455.

MAKRIDAKIS, S., WHEELWRIGHT, S.C. \& HYNDMAN, R.J. (1998) - Forecasting. Methods and Applications. Third Edition. John Wiley \& Sons. New York.

MENTZER, J.T. \& COX Jr., J.E. (1997) - Familiarity, application, and performance of sales forecasting techniques. Journal of Forecasting, v. 3, n. 1, p. 27-37.

PATTON, M. (1987) - How to Use Qualitative Methods in Evaluation, Sage Publications.

SANDERS, N.R. \& MANRODT, K.B. (1994) - Forecasting practices in US corporations: survey results. Interfaces, v. 24, n. 2, p. 92-101.

WEBBY, R. \& O'CONNOR, M. (1996) Judgement and Statistical Time Series Forecasting: a Review of the Literature. Int.J.Forecasting, 12, p.91-118.

WRIGHT, J.T.C. \& GIOVINAZZO, R.A. (2000) Delphi: uma Ferramenta de Apoio ao Planejamento Prospectivo. Cadernos de Pesquisas em Administração, v.1, n.12. 